\title{
CARDIOTHORACIC IMAGING
}

\section{Severe left atrium calcification (heart within a heart)}

Jessica Germino, MD, and Sudhakar N. J. Pipavath, MD

A 55-year-old man with a history of childhood rheumatic fever and subsequent rheumatic mitral stenosis after aortic and mitral valve replacement presented with abdominal pain and early satiety. On examination, his abdomen was tender to palpation, and he had lower extremity edema. Posteroanterior and lateral chest radiographs showed right pleural effusion, global cardiomegaly, and severe mural calcification of the left atrium (Figures 1-3). Right heart catheterization demonstrated a low cardiac index, moderately to markedly elevated pulmonary capillary wedge pressures, and severe pulmonary hypertension. His severe right heart failure was likely secondary to

From the Department of Radiology, University of Washington School of Medicine, Seattle, Wash.

Disclosures: Authors have nothing to disclose with regard to commercial support.

Received for publication Jan 12, 2014; accepted for publication Jan 13, 2014; available ahead of print Feb 14, 2014.

Address for reprints: Sudhakar N. J. Pipavath, MD, Department of Radiology, University of Washington School of Medicine, 1959 NE Pacific St, \#357115, Seattle, WA 98195 (E-mail: snjp@u.washington.edu).

J Thorac Cardiovasc Surg 2014;148:733-4

$0022-5223 / \$ 36.00$

Copyright (C) 2014 by The American Association for Thoracic Surgery

http://dx.doi.org/10.1016/j.jtcvs.2014.01.011

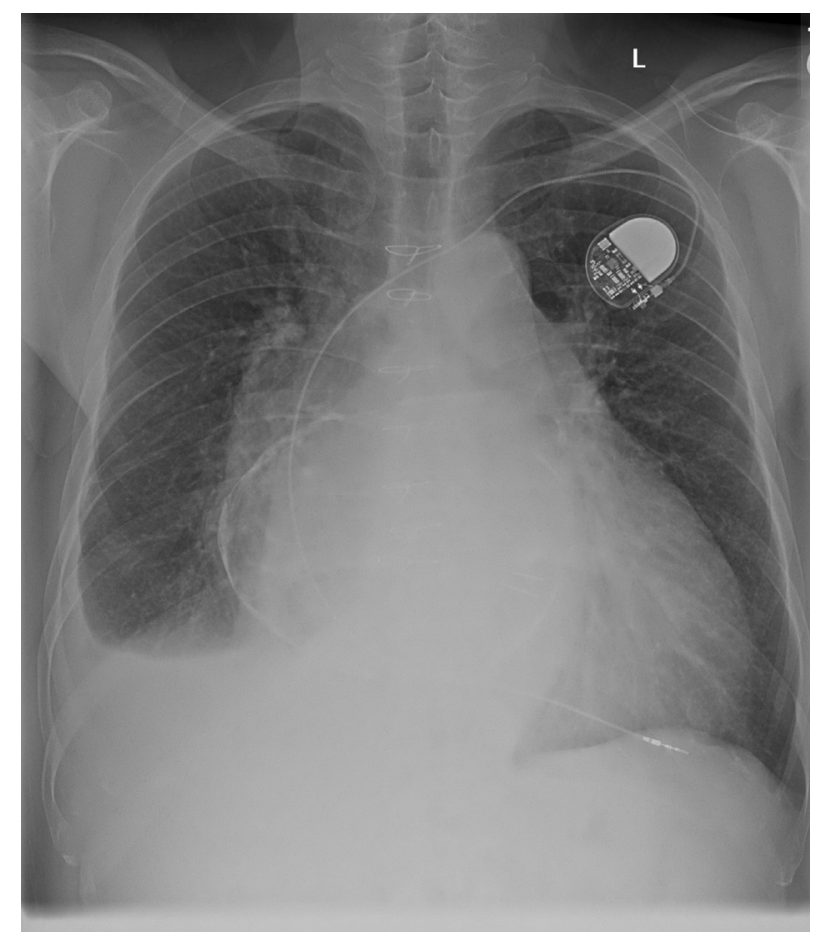

FIGURE 1. Frontal radiograph of the chest demonstrating right pleural effusion, global cardiomegaly, and severe mural calcification of the left atrium.

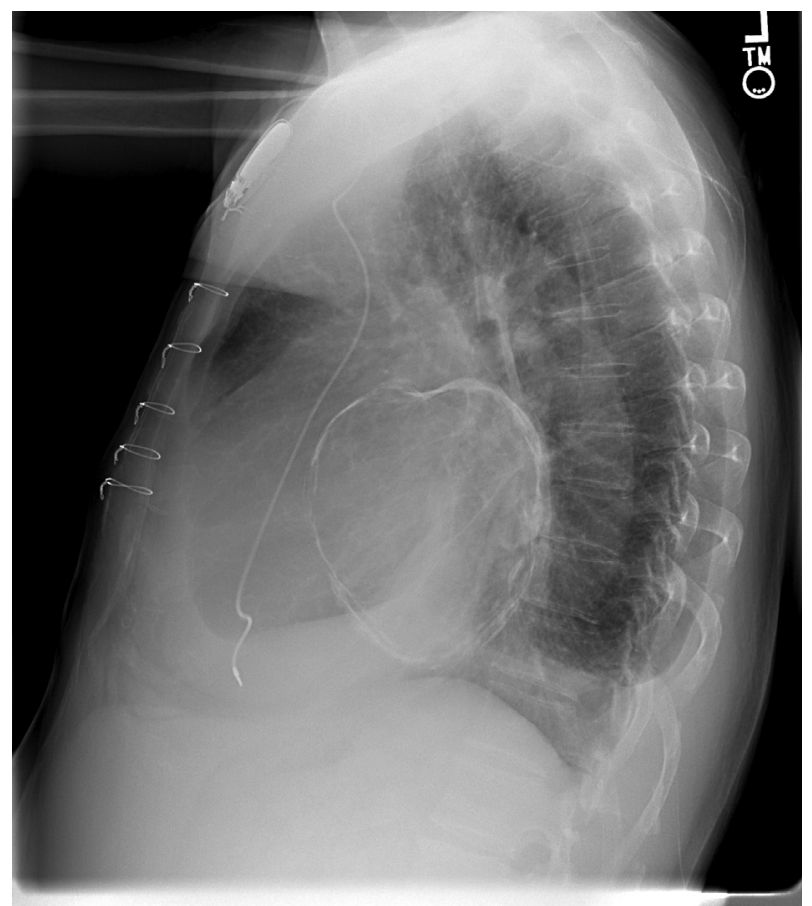

FIGURE 2. Lateral radiograph of the chest demonstrating extensive mural calcification of the left atrium.

pulmonary hypertension, which had developed in the setting of longstanding rheumatic mitral valve disease.

Severe calcification of the left atrium has been most commonly associated with rheumatic heart disease. ${ }^{1,2}$

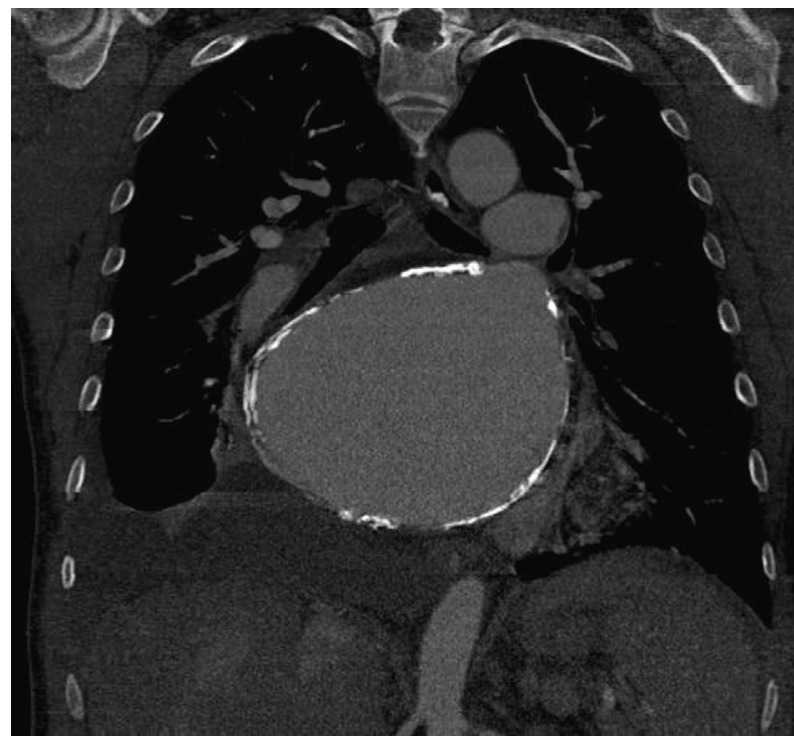

FIGURE 3. Coronal computed tomography scan of the chest demonstrating left atrial enlargement and calcification of the wall. 
Some have suggested this radiographic finding will be more frequently seen in patients with chronic rheumatic heart disease who have undergone previous mitral valve surgery. ${ }^{3}$ The published data have variably termed such diffuse left atrial calcification as a "coconut" or "porcelain" atrium. ${ }^{1,3}$ Clinically, this finding is of importance at mitral valve replacement. Traditional valve replacement has been complicated by difficulty suturing the left atrial wall and intraoperative risks of particulate embolization and hemorrhage. ${ }^{1,2}$ Thus, specialized surgical techniques, such as total endoatriectomy of the left atrium or calcium core debridement with valve replacement, have been alternatively used. ${ }^{4}$

\section{References}

1. Del Campo C, Weinstein P, Kunnelis C, DiStefano P, Ebers GM. Coconut atrium: transmural calcification of the entire left atrium. Tex Heart Inst J. 2000;27:49-51.

2. Santini F, Peranzoni P, Mazzucco A. Mitral valve replacement associated with massive left atrial calcification. Ann Thorac Surg. 1998;65:1456-8.

3. Funada A, Kanzaki H, Kanzaki S, Takahama H, Amaki M, Hasegawa T, et al. Coconut left atrium. Int J Cardiol. 2012;154:e42-4.

4. Nomura A, Fukuda I, Daitoku K, Fukui K. Enucleation of calcium core and in-situ valve replacement for massive posterior mitral annular calcification. Interact Cardiovasc Thorac Surg. 2011;12:652-4. 